\title{
Nourrir les vivants par la grâce des saints
}

La nourriture et le sacré dans le chiisme iranien

\section{Feed the Living by the Grace of the Saints}

\author{
Food and Sacredness in Iranian Shiism
}

Sepideh Parsapajouh

Résumé : En Iran, le don de nourriture pour une intention religieuse est un acte très répandu chez les croyants chiites, que l'on peut observer quotidiennement en ville comme à la campagne, dans les milieux aisés comme dans les milieux populaires. Pour comprendre les vertus sociales, matérielles et spirituelles d'une telle nourriture dans la vie pratique et la vision du monde des pieux chiites, cet article propose d'analyser le processus de préparation et de sacralisation de cette nourriture, et d'étudier quelques occasions importantes de don de nourriture votive dans la vie des croyants. Les données de cet article proviennent de recherches précédemment effectuées dans les milieux populaires iraniens, dans quelques sanctuaires chiites et au cimetière de Behesht Zahra de Téhéran, ainsi que d’entretiens réalisés à cette fin précise.

Mots clés : Don/contre don, imams impeccables, it'âm, nazr, nourriture votive, offrande aux morts, pur et impur

\footnotetext{
Abstract: In Iran, the giving of food for a religious purpose is a widespread act among Shiite believers, which can be observed daily in the city and in the villages, in both affluent and popular milieus. In order to understand the social, material and spiritual virtues of such food in the everyday life and worldview of Shiite devotees, this article proposes to analyse the process of preparation and sacredness of such food, and to study some important occasions of votive food giving in the lives of believers. The information in this article comes from previous research carried out in Iranian popular milieus, in some Shiite shrines and at the Behesht
} 
Zahra cemetery in Tehran, as well as from interviews conducted for this specific purpose.

Keywords: Gift/counter gift, impeccable imams, itâm, nazr, pure and impure, votive food, offering to the dead

Le 28 safar $^{1}$ de chaque année, ma mère préparait une marmite de sholeh-zard ${ }^{2}$ dont lodeur de safran et d'eau de rose embaumait toute la maison; vers le début de l'après-midi, l'œuvre finie, nous l'aidions à remplir des dizaines de bols de tailles diverses. Puis, nous dessinions sur la surface de chacun un motif à notre goût avec de la cannelle, un peu de pistache ou des pétales de roses. Nous sortions alors avec allégresse pour frapper à toutes les portes de la rue, tendre un bol à chaque personne en disant : "Pour vous! Cest le nazr de ma mère », et entendre en retour : «Que ce soit agréé » (qabul bâsheh), "Que Dieu l'accepte " (khodâ qabul koneh). Ce petit rituel familial et local faisait partie intégrante de notre calendrier, comme une évidence. Jamais je n’ai demandé à ma mère pourquoi elle faisait cela, si cétait pour un remerciement ou dans l'attente de quelque chose, et pourquoi à ce jour précis de l'année? Aujourd'hui encore, vivant à Paris depuis de longues années, le 28 safar n'est jamais un jour ordinaire pour moi ; il marrive de faire du sholeh-zard et même d'en distribuer à mes voisins, sans trop savoir quoi leur dire. Sorti du contexte chiite iranien, cet acte n'a plus de sens, sinon celui d'une réactivation de quelques souvenirs du passé.

En Iran, le don de nourriture pour une intention religieuse est un acte très répandu chez les croyants chiites, que l'on peut observer quotidiennement en ville comme à la campagne, dans les milieux aisés comme dans les milieux populaires. On appelle ce repas ghazâ-ye nazri (nourriture votive); il est fait à diverses occasions et donné à des gens de connaissance comme à des inconnus, en priorité des gens dans le besoin. Il peut être salé comme un bol de âsh (soupe épaisse avec des nouilles, des légumes secs et une sauce à base de yaourt), de halim (soupe épaisse à base de blé, avec de la viande), ou une assiette de chelo-khoresht (du riz et de la sauce avec des petits morceaux de viande et un peu de légumes); il peut être sucré comme du shir-berenj (riz au lait), du fereni (crème à base de farine de riz), du sholeh-zard (riz au safran); il peut s'agir tout simplement d'eau fraiche, d'une miche de pain, de quelques fruits, d'une poignée de raisins secs (keshmesh) ou de sucreries (noql). Cette nourriture est préparée et distribuée très souvent au nom-ou en l'honneurde l'un des quatorze Impeccables (chârdah ma'sum) du chiisme, souvent pour le remercier de l'exaucement d'un vœu qui lui est attribué ${ }^{3}$. Selon la croyance, l'intervention ou la présence du saint, à travers ne serait-ce qu'une bouchée ou une gorgée, suffit à transmettre et diffuser la grâce de Dieu (khodâ) dans 
le corps. Le bien produit ainsi aura un double effet : d'un côté, sur le corps et l'esprit de celui qui consomme le plat votif (nazri), et de l'autre, sur l'être et le destin de celui qui l'a offert. On a ainsi affaire à un système d'échange triangulaire dont la base, terrestre, relie horizontalement les hommes, et dont le sommet céleste est en Dieu, les saints imams assurant la circulation entre la base et le sommet.

Ces pratiques de dons de nourriture votive, et les liens horizontaux et verticaux qui se forment autour d'elles, prennent une ampleur bien plus importante lors doccasions religieuses particulières, comme pendant les moments d'épreuves de la vie individuelle et sociale.

Lannée où sécrivent ces pages, les mois sacrés de sha'bân ${ }^{4}$ et de ramadan coïncident avec l'épidémie de Covid19 à laquelle est confronté le monde-et l'Iran en particulier. Les chiites iraniens nen sont que plus motivés pour procéder à des offres de nourritures votives. Mais par mesure d'hygiène, et pour prévenir justement les effets d'une telle ferveur, les autorités religieuses et étatiques ont formellement interdit tout partage public de nourriture préparée. Un discours officiel ne cesse de conseiller le remplacement du don de nourriture préparée par un don d'ingrédients secs ou d'argent. Diverses initiatives se sont généralisées, aux échelles locales et nationales, en vue d’apporter aide et protection aux ouvriers journaliers, aux vendeurs à la sauvette, à tous ceux qui ont perdu leur emploi, mais aussi aux personnels soignants des hôpitaux. Cependant, le fait de ne pas pouvoir préparer à manger et nourrir les autres de ses propres mains est vécu comme une privation éprouvante par toute une partie de la population, coutumière de cette pratique. Que recèle donc cette nourriture votive (nazri) chez les croyants chiites pour que son abandon soit aussi difficile? Pour comprendre les vertus sociales, matérielles et spirituelles d'une telle nourriture dans la vie pratique et la vision du monde des pieux chiites, nous commencerons par analyser le processus de préparation et de sacralisation de cette nourriture. Puis, nous étudierons quelques occasions importantes de don de nourriture votive dans la vie des croyants.

Les données de cet article proviennent de plusieurs recherches effectuées sur d'autres thématiques, dans les milieux populaires des villes iraniennes de Karaj et de Téhéran, ainsi que dans quelques sanctuaires chiites et au grand cimetière de Téhéran (Behesht Zahra) (Parsapajouh 2016, 2019, 2020). Des entretiens ont récemment été réalisés par WhatsApp pour compléter ces données de base. Soulignons que notre échantillon n'entend pas être représentatif de toute la population iranienne, mais d'une grande partie de la société concernée par la foi et la pratique religieuse.

\section{Lacte de nourrir, un geste sacré}

Si la nourriture a une valeur symbolique et sacrée en Iran chiite, c'est dans l'acte de nourrir les autres, et non dans celui de se nourrir. De très nombreux hadiths 
chiites, récits hagiographiques et dictons populaires, transmis oralement de génération en génération et à travers tous les milieux sociaux, louent l'acte de nourrir les "serviteurs de Dieu » (bandegân-e khodâ), c'est-à-dire tout simplement « les gens ». Le terme religieux employé en arabe pour désigner la valeur de cet acte est itâm (acte de nourrir), mais la notion est souvent intégrée dans celle, plus large, d'infâq (don). Ces deux termes sont coraniques, extraits de versets que les pieux chiites connaissent sous ces noms. Ainsi, les deux versets coraniques $2: 274$ et $3: 92$ sont connus comme les versets de l'infâq; et le verset $76: 8$ qui, selon la croyance et l'exégèse chiites, fut révélé pour l'imam Ali et son épouse Fâtemeh, est connu sous le nom de verset de l'it'âm.

Au-delà du degré de croyance ou de piété religieuse, la coutume de donner ou de partager la nourriture marque les rapports quotidiens entre Iraniens de toutes catégories socio-économiques. En donnant à manger aux autres, la personne gagne certainement en prestige social ${ }^{5}$. Pourtant, il est manifeste que ce qui la motive profondément est plus subtil. En préparant à manger, la personne se demande souvent qui sera nourri de ce repas en plus des bénéficiaires prévus, quelle "part» (qesmat) est à prévoir pour l'inconnu et, par conséquent, elle en fait toujours « un peu plus » que ce qui aurait été initialement prévu. Il existe en outre une croyance assez souple et extensible concernant la "part quotidienne " (rûzi) de tout un chacun. Rûzi (de rûz : jour) est l'appellation des provisions, matérielles ou immatérielles, pourvues chaque jour par Dieu à son serviteur, de manière imprévisible, par l'intermédiaire d'autres. L'on croit donc que dans tout ce que l'on cuisine réside une part en excès qui cherche son "maître " (sâheb). Si elle le trouve, l'honneur revient à la personne qui a cuisiné, dans l'idée que Dieu l'a choisie comme son intermédiaire pour transmettre le rûzi destiné à ce serviteur. C'est pourquoi le chef de famille et la maîtresse de maison, selon la répartition traditionnelle des rôles au sein de la famille iranienne, sont généralement attentifs aux gens qui les entourent, voisins, membres de la famille, amis et même inconnus, susceptibles de recevoir une part de leur nourriture ; et l'on voit couramment les gens s'inviter mutuellement avec force insistance. L'« invité non appelé» (mehmân-e nâ-khândeh) est encore plus respecté que les autres, il est appelé l'" ami de Dieu » (habib-e khodâ). Dans ce système de valeurs centré sur le don, celui à qui on offre de la nourriture n'est pas censé la refuser ; lobligation de donner s'accompagne de l'obligation de recevoir, conforme au triangle maussien dans son Essai sur le don. Ne pas refuser " la main » de quelqu'un fait partie des premières règles de bienséance qu'une mère apprend à son enfant : « Ne refuse jamais la main de quelqu'un! Prends de ce qu'on te donne, ne serait-ce qu'un tout petit peu ». De même, les enfants apprennent dès leur plus jeune âge à partager ce qu'ils mangent avec les autres ; en toutes circonstances-les transports en commun, les espaces publics -, qui s'apprête à mettre quelque chose dans sa bouche en propose d'abord une part à ceux qui se trouvent à côté de lui, connus ou inconnus, en disant : «be-farmầid» (servez-vous). Cependant, au sein de cet univers culturel, certaines occasions donnent lieu à des pratiques 
nourricières plus spectaculaires, toujours liées au sacré, que nous allons maintenant examiner.

\section{La nourriture comme un pacte divin : le nazr}

Le terme arabe nadhr, prononcé en persan nazr et couramment utilisé en Iran, peut se traduire par «l'acte de consacrer quelque chose à Dieu » (Kazimirski) ou par « vœu, action de vouer, ex-voto » (Lazard). Il s'agit ici aussi d'un terme coranique, assez chargé et ambigu, apparaissant à cinq reprises dans le Coran $(2: 270 ; 3: 35 ; 19: 26 ; 22: 29 ; 76: 7)$. Sans prétention d'interpréter le sens du texte coranique, indiquons seulement que le terme nazr, dans tous ces versets, se réfère à une forme de pacte avec Dieu, mais sans lien avec l'alimentation, sauf peut-être dans le verset $2: 270$, où il apparaît avec le terme infâq (don), interprété par des croyants comme signifiant un don de nourriture. Dans le dictionnaire persan unilingue de Dehkhodâ, nazr est défini comme un pacte qu'un homme noue volontairement avec Dieu, la contrainte qu'il se donne de faire quelque chose pour contenter Dieu.

Les acteurs du nazr sont en premier lieu les femmes au sein de l'entité familiale, mais aussi les hommes quand cette pratique prend, comme nous le verrons, une dimension plus large et institutionnelle. Dans les deux cas, les enfants, les autres membres de la famille ou du groupe participent aussi volontiers à la préparation du nazr, même avec la plus petite contribution (une petite somme, un ingrédient aussi basique que le sel, un petit geste comme remuer). Tous ceux qui participent d'une manière ou d'une autre à la préparation de ce repas votif sont censés recevoir leur part de récompense pour cette œuvre pieuse.

Ces nazr sont souvent conditionnels ${ }^{6}$. On noue un pacte avec lêtre transcendant (Dieu ou l'imâm) dans le cadre d'une transaction : on lui demande quelque chose (la guérison, la conception, une réussite, ou la résolution d'un problème) et on lui promet de le rendre, si le vœu est exaucé, sous forme d'une bonne action en direction des autres humains. Ce que l'on doit rendre est défini dans le "pacte » et ne devient obligatoire que si le résultat souhaité est obtenu. Mais l'on peut aussi le devancer afin d'assurer davantage la réalisation du vœu. Ce que lon formule comme obligation de retour peut être une bonne action spirituelle ou un don matériel. Sous forme spirituelle, ce retour peut consister, par exemple, à réciter un nombre précis de formules pieuses, de prières, de sourates coraniques, ou à faire un effort considérable pour la mise en épreuve du corps. Sous sa forme matérielle la plus visible, le nazr se traduit par la distribution de nourriture à certaines occasions sacrées du calendrier religieux ${ }^{7}$. C’est ce genre de nazr que nous avons évoqué dans l'introduction de cet article. Là, le croyant peut s'engager dans la préparation d'un repas plus ou moins compliqué en quantité précise, ou dans la distribution d'une certaine quantité de boisson fraiche ou chaude. La destination de ces aliments votifs 
peut être définie : les pauvres, les pèlerins d'un certain lieu saint, les habitués d'une certaine mosquée, les endeuillés (azâdâran) d'un certain hoseyniyeh ${ }^{8}$, les participants de telle ou telle réunion religieuse. Mais la destination peut également rester indéterminée. Ainsi plus nombreux sont ceux qui peuvent en recevoir une part : collègues, clients, amis, voisins, familles, et jusqu'aux passants dans la rue ${ }^{9}$.

\section{L'impératif de pureté dans la préparation du repas}

Dans la préparation de la nourriture votive, la notion de pureté (tahârat) tient une place fondamentale. La distinction du pur et de l'impur, en particulier dans l'alimentation, est présente dans la plupart des religions, et généralement en islam. Elle est particulièrement importante dans le chiisme iranien, peut-être en raison de son ancrage dans le zoroastrisme, la religion de l'Iran préislamique. Irréductible à la distinction juridique, objective, du licite (halâl) et de l'illicite (harâm), elle possède une dimension mystérieuse, subjective et magique. Elle détermine toute la vie domestique de la famille iranienne, à commencer par les pratiques culinaires ${ }^{10}$.

Selon des croyances vivaces imprégnant les manières de vivre des pieuses familles iraniennes, la pureté de la nourriture dépend de deux facteurs indépendants : d'un côté, l'intégrité de l'argent gagné pour acheter ses ingrédients ; de l'autre, la rigueur du processus de préparation. Si l'islam est dès l'origine une société commerçante qui ne méprise pas les liens économiques, l'argent n’en est pas moins fondamentalement considéré comme impur et potentiellement mauvais, en ce qu'il attache l'homme à la vie d'ici-bas et à ses plaisirs éphémères, toutes choses dont il faut se détacher pour gagner la vie dernière. Il n'est a priori pas immoral de gagner de l'argent, mais il est obligatoire d'en donner pour légitimer la fortune acquise. Autrement dit, plus on donne de l'argent que l'on gagne, plus on purifie la somme restante. Ainsi, l'argent de celui qui ne paye pas le khoms, l'aumône prescrite dans le chiisme, correspondant à un cinquième du restant du revenu annuel, est considéré comme impur. Largent acquis au moyen de l'usure ou d'activités illégales l'est également : non seulement des actes de charité financés par cet argent ne suffisent pas à le purifier, mais ils propagent son impureté comme une maladie. Seul l'argent honnêtement gagné est considéré assez pur pour être utilisé dans des dons consacrés comme des dons de nourriture. Les témoignages suivants montrent l'ampleur de cette croyance dans l'esprit du fidèle :

"Beaucoup de problèmes physiques et moraux sont causés par la nourriture impure que l'on mange, une nourriture issue d'une fortune impure (mâl-e nâââk) et polluée (âludeh). Comment peut-on manger l'argent volé ou tiré de la souffrance des autres ?! (femme, 51 ans, Téhéran). 
«Dans notre maison de charité (kheyriyyeh), parmi les sommes que les gens nous donnent, nous mettons d'un côté l'argent de ceux que nous savons être pieux et honnêtes pour acheter la nourriture des pauvres avec lui seulement. Nous n'utilisons pas n'importe quel argent pour acheter cette nourriture. Nous sommes responsables de ce que nous donnons à manger aux autres » (femme, 49 ans, Téhéran).

"Ce qui nourrit notre corps est aussi important que ce qui nourrit notre esprit, il faut faire très attention. Dans ma famille, on croit à la bouchée licite (loqmeh-ye halâl). Même quand on m'invite aux repas entre collègues, je me retiens, je refuse tant que je peux, je trouve un prétexte pour m'excuser. Quand je suis obligée, (ce qui peut arriver), alors je mets de côté l'équivalent du prix de ce repas pour le rajouter à mes dons mensuels (vujuhât-e mahâneh)» (femme, 45 ans, Téhéran).

La pureté de la nourriture est assurée d'autre part par le processus de sa préparation. En premier lieu, les ingrédients, puis la personne qui cuisine, doivent être propres. Cette propreté, chez les plus croyants, n'est pas seulement liée à une considération hygiénique, mais à une notion d'état de pureté rituelle. Ainsi, la viande, les légumes frais et secs, le riz, comme tous les ustensiles de cuisine, doivent être rincés à grande eau pour garantir lélimination de toute particule d'impureté, selon le droit canonique, lié au fiqh.

Dans la préparation des repas, notamment des repas votifs, la propreté ou la pureté de celle ou de celui qui cuisine est assurée par des ablutions rituelles, semblables à celles exigées pour la prière.

« Avant de préparer à manger, je fais les ablutions, je ne touche jamais les ingrédients sans vuzu (petite ablution), même si je suis dans la période de menstruation » (femme, 45 ans, Téhéran).

« Je pense que si vous dites aux Français que nous, les chiites, ne touchons pas à un repas qui a été fait par un jonob, ils vont être choqués, parce que je pense qu'ils ne savent même pas ce que c'est que le ghosl-e jenâbat ${ }^{11}$ (rire) " (femme, 49 ans, Téhéran).

En réponse à mes questions : "Mangez-vous la nourriture qu’on vous donne? Acceptez-vous les invitations des gens ? ", les réponses de mes deux interlocutrices sont intéressantes :

"Si c'est un nazri [repas issu d'un nazr] oui ! Mais si c'est à l'occasion d'une réception et que j’ai des doutes au sujet de la source des revenus des hôtes, non, je refuse. En même temps, comme je ne veux pas décevoir celui qui m'invite, je dis que je refuse en général d'aller aux festins luxueux, ce qui est vrai. J’entretiens les relations avec les gens de ma famille à cause du lien de parenté (seleh-ye rahem), je rends surtout visite à ceux qui sont malades, mais sinon, j'essaie de garder ma distance avec ceux que je ne connais pas bien. Parce que je ne sais pas s'ils respectent les règles religieuses ou pas. Avec tout le respect que jai pour les gens, si je ne suis pas sûre que leur nourriture est pure, je n’en prends pas » (femme, 51 ans, Téhéran). 
« Je fais très attention. Parce qu’il ne faut pas gâcher, par un simple désir, tout ce qu'on essaie de gagner avec beaucoup d'effort spirituellement. Pourtant je ne pense pas être très exigeante. Vous savez, les gens très pieux et certains ulémas sont très susceptibles. Ils ne mangent jamais de la nourriture dont ils ne connaissent pas la source. Par exemple, même le pain, ils vont le chercher dans des boulangeries dont ils sont sûrs que le boulanger le prépare après avoir fait ses ablutions, et que celui qui touche le pain ne touche pas l'argent : pour eux, même toucher la monnaie est makrûh (répréhensible)»(femme, 45 ans, Téhéran).

Cependant, une autre interlocutrice, peut-être consciente que ses paroles seront rapportées en Occident, insiste sur la tolérance interreligieuse afin de nuancer son propos :

«Toutefois, il faut dire aussi que la nourriture des gens du Livre (ahl-e ketâb) est tout à fait pure. Même si lon ne peut pas manger de la chair illicite [du porc], on devrait faire confiance à leur manière de faire à manger, parce qu'ils le font selon leur propre principe de pureté. C'est l'avis du Guide suprême, et c'est ce qu'il fait quand il va rendre visite aux familles de martyrs chrétiens et juifs » (femme, 49 ans, Téhéran).

Lors de la préparation, de nombreuses formules pieuses-prières, versets coraniques, bénédictions adressées à l'imam-sont formulées et parfois soufflées dans le repas, tandis qu'on le remue ou qu'on y ajoute un ingrédient. Ces formules pieuses confiées au repas sont censées pour partie y rester et pour partie monter au ciel, avec la vapeur et la fumée. Lobservance des règles de pureté, tant physiques que morales, conditionne doublement la réussite du processus.

Toutes ces pratiques font de la préparation d'un nazr un véritable acte cultuel visant une communication avec le monde céleste. Le respect des règles et la pureté de l'intention sont censés conférer au repas un pouvoir quasimagique : quand le saint imam agrée le repas préparé et offert en son nom, sa sainteté se transmet à la nourriture, dont la vertu agit aussi bien sur l'âme que sur le corps de ceux qui la consomment ; le bien physique et moral ainsi produit satisfait l'imam qui, en retour, ne manque pas de récompenser ses auteurs. Une forme déchange s'instaure ainsi entre les croyants de ce monde et les imams appartenant au monde dit supérieur (âlam-e bâlâ).

\section{S'asseoir autour du sofreh du saint}

La nourriture est aussi associée à la notion de sofreh. Le terme est communément traduit en français par "nappe », mais le sens de sofreh est bien plus chargé et noble. La notion englobe diverses valeurs morales comme le partage, la générosité, l'hospitalité, mais aussi et surtout la baraka. Différentes expressions persanes sont composées sur ce mot : sofreh-dari (détenir un sofreh) désigne la générosité ; ham-sofreh-gi (ham : co-) signifie une amitié solide ; 
"s'asseoir autour du sofreh de quelqu'un » signifie être entré dans sa confiance ; sofreh-ye bâz (nappe ouverte) signifie la clémence et l'altruisme. Un proverbe dit : " si tu veux connaître quelqu'un, partage le sofreh avec lui ». Enfin et surtout, pour désigner la haute qualité morale de quelqu'un qui prépare des repas votifs pour les imams, on dit : sofreh-dâr-e hazrat ast : « il est le détenteur de la nappe du saint $»$.

Traditionnellement, les Iraniens s'installent à même le sol autour d'une nappe pour manger; seules les familles les plus modernes ou occidentalisées en ont plus ou moins perdu l'habitude. Il s'agit d'une composition qui, à l'instar de la «table » française, possède de strictes règles de bienséance. Mais la caractéristique première du sofreh, qui la distingue de la table, est qu'il peut toujours sagrandir, tant que le sol le permet, pour recevoir davantage de convives, et c'est là le sens de la baraka d'un sofreh. Le sofreh nest pas un simple outil du manger, il représente en soi une obligation sacrée : il faut toujours s'asseoir autour d'un sofreh pour manger; on ne mange pas debout, ni seul.

" Je me rappelle, même sur le front de guerre, même dans les tranchées, on se mettait tous autour d'un sofreh. C'étaient nos keffiehs qui en faisaient fonction! » (homme, 65 ans, Téhéran).

«Mon père, aussi loin que je me souvienne, a toujours fait le nazr d'offrir le repas de rupture du jeûne [du Ramadan] la nuit de la naissance de l'imam Hasan. On étend un très grand sofreh à la maison, et toute la soirée, la porte de la maison est ouverte à tous. Tous les gens du quartier, et même d'autres quartiers, viennent groupe par groupe, sans arrêt jusquà la fin de la soirée, manger autour du sofreh de l'imam Hasan » (homme, 43 ans, Ahvâz).

Selon la croyance, le repas dédié à un imam accroît, par un pouvoir magique, la baraka du sofreh: la quantité du repas augmente et il peut nourrir toujours plus de personnes que prévu. C'est pourquoi traditionnellement, il y a une réticence à déterminer à l’avance le nombre de portions.

"L’année dernière, le nazr de mon père a coïncidé avec l'horrible inondation qui avait ravagé les villages d'alentour. Nous lui avons proposé, au lieu de donner à manger aux gens du quartier, de faire des portions individuelles et de les apporter aux sinistrés de ces villages. Il a accepté l'idée, mais pas la méthode. Il a dit : «Faire des portions ?! Le repas de l'imam Hasan est porteur de baraka! Je ne ferai quétendre une nappe à son nom ». Alors, nous avons apporté toutes les marmites et tous les ustensiles dans la mosquée d'un de ces villages, et nous avons étendu le sofreh comme à la maison... » (homme, 43 ans, Ahvâz).

"Un sofreh étendu au nom d'un imam porte beaucoup de baraka, ça oui !... Vous savez, dans les hadiths des imams, on dit que des anges viennent sasseoir autour d'un sofreh dédié à un imam » (homme, 65 ans, Téhéran). 


\section{Les occasions du nazr dans le temps et dans l'espace}

Les occasions de préparation des repas dédiés aux imams sont nombreuses et très diverses au cours de l'année. Les formes et la quantité de ces offrandes sont aussi multiples. Cela peut aller d'un petit repas qu'une mère de famille prépare pour sa propre maison, à des quantités plus importantes qu'elle distribuera parmi les amis et voisins à une occasion particulière (souvent l'anniversaire de la naissance ou de la mort d'un imam), jusqu'aux repas collectifs préparés par des hommes au sein d'un groupe pour une cérémonie religieuse avec de nombreux fidèles.

Selon des croyances souples devenues coutumières au sein des familles pratiquantes, chaque jour de la semaine est attribué à un ou plusieurs des " quatorze Impeccables». Dans le temps ordinaire, en l'absence d'occasion religieuse particulière, le repas de chaque jour peut se préparer dans l'intention des saints imams du jour, auxquels peuvent être ajoutés, selon létat du cœur de la cuisinière ou du cuisinier, d'autres figures sacrées : des enfants des imams, des « amis de Dieu » (awliyâ), des martyrs, et même des ancêtres.

Outre ces attributions quotidiennes, le calendrier chiite est parsemé d'occasions liées à l'histoire des saints imams, de leurs enfants, petits-enfants et compagnons (Soroushfar 2020) : anniversaires de morts et de naissances, épisodes liés aux quatre mois sacrés de muharram, safar, rajab et sha'ban, le jour de la première révélation du Prophète, les nuits de la destinée (qadr), lévénement de Ghadîr ${ }^{12}$, le jour de 'arafa' ${ }^{13}$, le jour de eyd-e qurbân, le jour de eyd-e fitr, etc. Chacune de ces occasions peut motiver un nazr de nourriture. La croyante ou le croyant les choisit selon son rapport intime avec les imams et le sacré. Ainsi, au sein de familles croyantes, on trouve souvent des traditions plus ou moins anciennes de faire des nazrs pour certains saints à des dates précises.

Lors de trois occasions particulières, la préparation de cette nourriture votive est plus spectaculaire que d'habitude. La première, et la plus importante, est liée aux célèbres cérémonies de commémoration du martyre de l'imam Hoseyn (à Karbala en 60/681) qui commencent avec le début du mois de moharram et continuent jusqu'au quarantième jour suivant la date du martyre, appelé Arba'în, situé au mois suivant, safar. Durant cette période qui couvre près de cinquante jours de l'année, les dix premiers jours, et plus particulièrement les neuvième (Tâsu'â) et dixième jour ('Ashurâ), puis le jour de Arba'în, les préparations de nourriture votive atteignent leur comble pour occuper largement l'espace public de toute ville et de tout village. On assiste à des sacrifices d'animaux et à des distributions de nourriture dans divers lieux : hoseyniyeh, mosquées, maisons, ainsi que des stands éphémères aux coins des rues. D’autres espaces sont consacrés aux préparations collectives dans de grandes marmites, constamment entourées de foule. Ces repas sont surtout préparés pour les fidèles participant aux performances cérémonielles d'auto-mortification, mais tout un chacun peut en bénéficier à satiété (figure 1). On en apporte toujours aux malades, aux vieillards, aux 


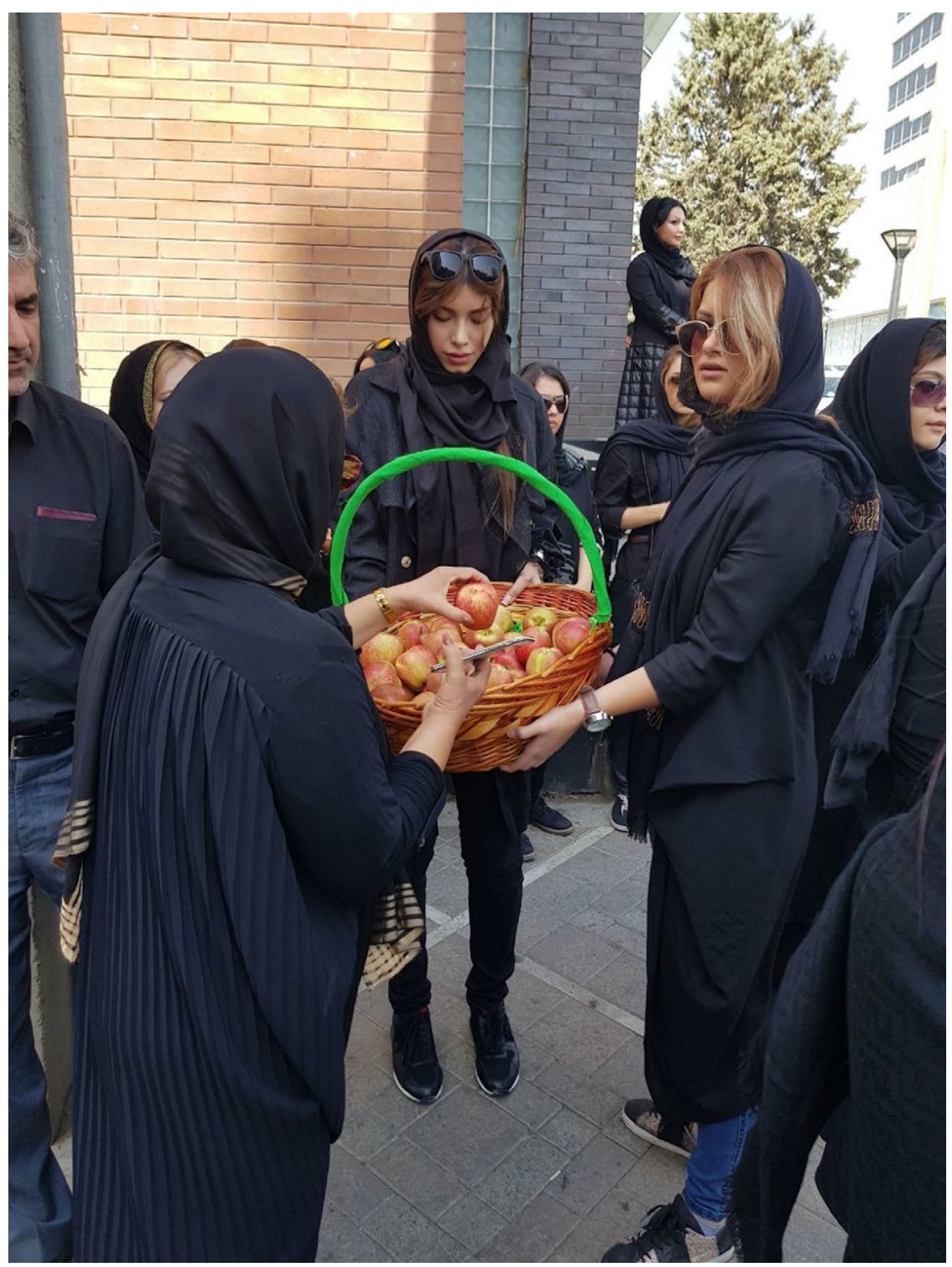

Figure 1 : Don de pommes lors de la cérémonie de 'Ashurâ à Téhéran (c) Jalal Rafifar, $2017^{14}$ 
femmes enceintes et aux enfants (Vivier 2006 ; Bromberger 2013 : 184-185; Parsapajouh 2016 : 267-310, au sujet de Moharram et Neuve-Eglise 2020 au sujet de Arba'în).

La deuxième occasion importante du don de nourriture votive au nom d'un saint imam est liée à la pratique de la ziyârat (visite pieuse, pèlerinage). Selon la croyance chiite, de retour de sa visite pieuse au tombeau d'un imam, un pèlerin est porteur de la bénédiction et de la sacralité reçues de sa rencontre avec l'imam. Les membres de sa famille et ses amis sempressent de lui rendre visite; on le touche, on l'embrasse afin de bénéficier de sa baraka obtenue par la ziyârat. À son tour, le pèlerin les reçoit avec un repas appelé valimeh. Ce repas est considéré comme béni par la sacralité de l'imam ; il est particulièrement précieux quand le pèlerin revient des 'atabât (les sanctuaires des imams en Irak). Les invités en mangent autant qu'ils veulent et en rapportent aussi, même en quantité infime, pour ceux qui nont pas pu venir.

La troisième occasion de nazr, extrêmement valorisée, est temporelle mais surtout spatiale, liée au lieu même du saint. Au sein de certains sanctuaires comme celui de l'imam Reza à Mashhad, ou celui de Fâtemeh Ma'sumeh à Qom, tous deux en Iran, un repas collectif est préparé tous les jours par des fidèles bénévoles du sanctuaire, financé par les « revenus du saint »-issus principalement des dons des pèlerins-pour être distribué gratuitement parmi les pèlerins. Ce repas, appelé ghazâ-ye hazrat (« le repas du saint»), est particulièrement précieux et convoité par les pèlerins. Les sanctuaires disposent de grandes cantines, mais la demande étant largement supérieure à l’offre, ce repas gracieux n'est, de fait, pas facilement accessible. D'où l'existence de systèmes de distribution complexes, comme celui du repas du huitième imam au sanctuaire de Mashhad depuis 2018.

Les responsables de la cuisine du sanctuaire de l'imam Rezâ à Mashhad ont inventé une application téléchargeable sur smartphone (app.razavi.ir), permettant à chaque pèlerin de candidater à une part du repas du saint. Il doit s'inscrire sur cette application pour participer, cinq journées durant, à des tirages au sort ayant lieu toutes les dix minutes ${ }^{15}$. Un système équivalent a été mis en place au sanctuaire de Qom pour le repas de Sainte Fâtemeh Ma’sumeh ${ }^{16}$. Cependant, ce que nous avons pu observer plus brièvement au sanctuaire des dixième et onzième imams à Samarrâ, en Irak en été 2018, était tout aussi important, quoique plus traditionnel et moins rationalisé.

La viande fait partie intégrante de ces repas et provient toujours d'un sacrifice selon les règles religieuses. Cette viande est précieuse, on n'en sert qu'en petite quantité. La valeur de ces repas, on l'a compris, ne dépend pas de sa quantité, ni de sa qualité gustative, mais de sa sacralité ; c'est elle qui le rend abondant et délicieux pour tous les croyants qui en ont une part. 


\section{Nourrir les vivants, apaiser les morts}

Tout visiteur étranger d'un cimetière iranien serait frappé par la présence spectaculaire de quantités de nourriture : gâteaux, fruits, dattes, halva, boissons, etc. (figures 2 et 3). Il ne s'agit pas, comme dans d'autres cultures (par exemple Ruhlmann 2016, à propos de la Mongolie), d’offrir de la nourriture aux morts, mais bien de nourrir les vivants. Il est en effet de coutume, quand on visite la tombe d'un parent ou d'un proche, d'offrir de ces friandises citées aux autres visiteurs et aux passants. Les pauvres et les mendiants savent tous que c'est là, surtout les jeudis, qu'ils peuvent trouver de la nourriture distribuée avec générosité. L’on ne demande en échange qu'une prière ou une invocation pour le défunt, même à celui qui ne le connaît pas, qui ne sait ni son nom, ni son âge, ni son sexe. À la base de cette pratique, on trouve cette croyance : le fait de nourrir le vivant dans l'intention du défunt apaise lâme de celui-ci.

Cette croyance elle-même repose sur la conception du terrible univers du barzakh (terme coranique signifiant "barrière », ou tout ce qui sépare), intermédiaire entre la mort et la Résurrection finale (Terrier 2020). Tout individu, bon ou mauvais, à l'exception des saints et des martyrs, est censé demeurer dans ce monde dès après la mort et dans l'attente du Jugement dernier ; il y est conscient, sensible, et souffre toujours plus ou moins, puisque aucun homme



Figure 2 : Une boite de gâteaux posée sur la tombe parmi les fleurs. Téhéran, cimetière de Behesth Zahrâ, (c) S. Parsapajouh, 2017. 
ordinaire n'est parfait ni innocent. Si la mort est un événement redoutable, c'est aussi quaprès elle, la capacité d'action de l'homme est suspendue : il ne fait que subir les conséquences des actes qu'il a commis de son vivant. Mais il peut toujours recevoir ce que les vivants lui envoient de prières et de pensées ${ }^{17}$. Pour cette raison, les croyants se donnent le devoir de penser à leurs morts et

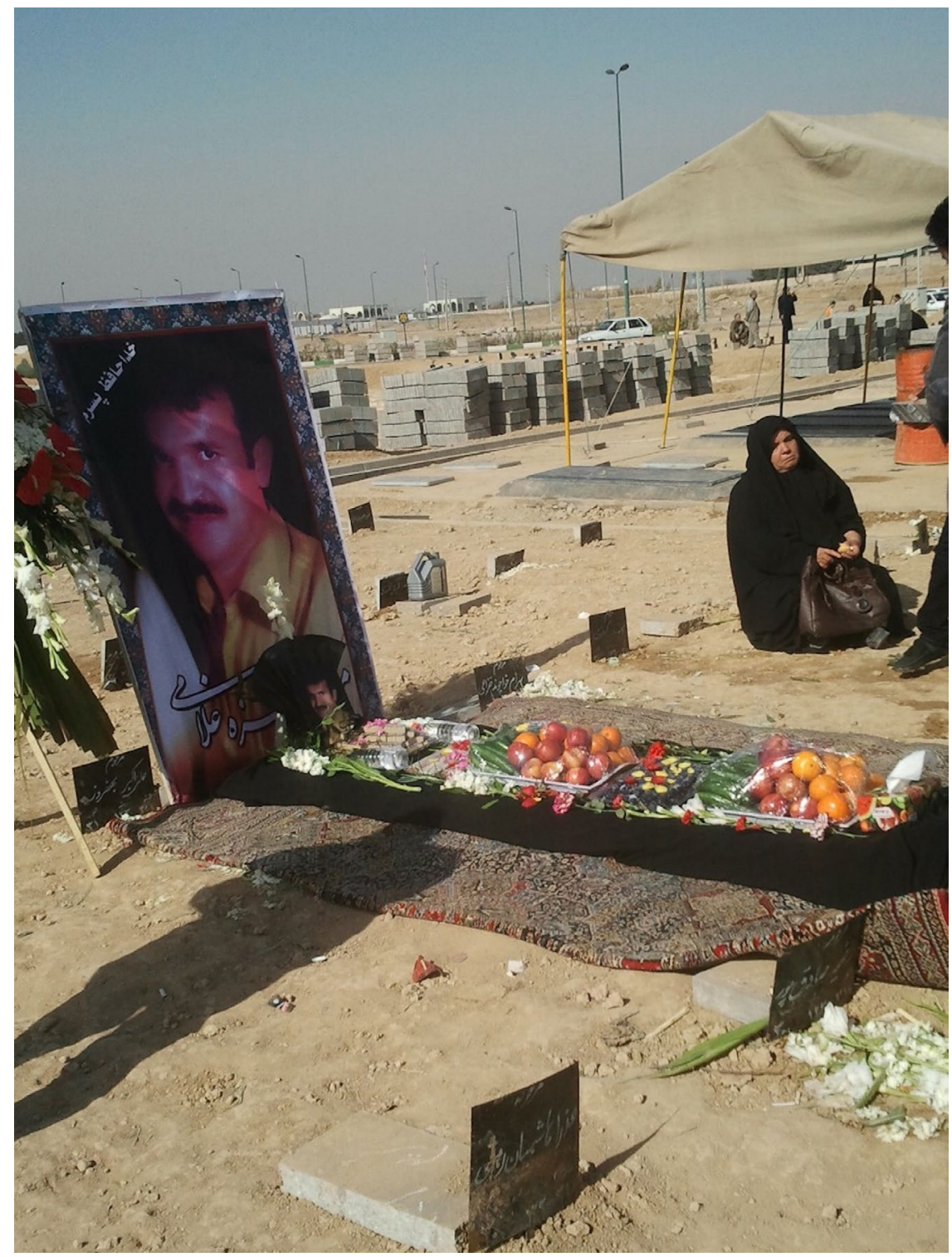

Figure 3 : Fruits, gâteaux et dattes, posés sur la tombe d'un défunt fraichement enterré à Behesth Zahra de Téhéran, () S. Parsapajouh, 2015. 
s'efforcent de leur envoyer, par leurs bonnes actions et leurs prières, de quoi les soulager autant que possible. Dans ce système de croyances, le fait de nourrir les vivants aidera lâme du mort, grâce aux prières que les nourris formuleront en retour pour lui. Ces actes de don et de charité, effectués au nom et dans l'intention des morts, sont nommés kheyrât (bontés, bienfaisances). Ils ne sont pas circonscrits aux espaces mortuaires mais peuvent occuper l'espace de la vie quotidienne. Le jeudi soir, dans les grandes avenues ou les bazars des villes, il nest pas rare de voir un plateau de dattes ou de gâteaux posé sur un tabouret, à disposition des passants. Ceux qui se servent savent qu'en retour, ils doivent formuler une petite prière, rien qu'un salavât (la formule pieuse de bénédiction du Prophète et de sa descendance), pour une personne décédée qu'ils ne connaissent pas ${ }^{18}$.

Toutefois, il faut préciser qu'autant les croyants ordinaires bénéficient très volontiers d'un nazr réalisé au nom d'un saint, autant ils auront une réticence à prendre une part du repas dédié à un mort et à la savourer. Lors des enterrements, certains, les plus pieux et rigoureux, préfèrent ne pas goûter au repas funèbre, parce qu'ils le considèrent chargé du chagrin et de la tristesse des endeuillés. Ce refus n'enlève rien à leur volonté de réciter les prières attendues en échange du repas. Ils le font même volontiers, convaincus que lâme du mort, une fois apaisée, formulera des prières à son tour pour ceux qui l'ont soulagée. On se retrouve encore dans le système de don et de contre-don.

\section{Conclusion}

La tradition religieuse et populaire du nazr, don de nourriture votif, en Iran, s'inscrit dans la croyance en un lien constant d'échange et de transaction entre les hommes et le divin par l'intermédiaire des personnes saintes que sont les imams, leurs parents et enfants, les martyrs et les ancêtres. Mais si le motif et la finalité du don se situent dans l'au-delà, c'est aussi un lien social horizontal, circulaire et proprement interminable, que le nazr illustre et nourrit. Le don est motivé soit par l'espoir de recevoir une récompense, soit par l'obligation de rendre ce que l'on a reçu (ou son équivalent) par la grâce d'un saint, mais à d'autres hommes ou par l'intermédiaire de ceux-ci. Dans cette "transaction " avec le divin, ce sont bien les humains que l'on nourrit ; et ceux-ci " rendent » ce qu'ils ont reçu du saint par l'intermédiaire de l'homme, ou de l'homme au nom du saint, sous forme de prières qui, montant au ciel, font redescendre encore plus de grâce sur les donateurs. Le nazr nest ni une simple pratique rituelle, ni un échange seulement social, mais un rapport d'interaction multilatérale entre les vivants et les saints. Ce rapport est constant, suscité par d'innombrables occasions liées au calendrier et à l'espace sacré des chiites. Sur le plan social, ces échanges sont extrêmement généralisés et englobants. Selon les dimensions du nazr, ils impliquent tantôt des femmes et des enfants (à léchelle de la maisonnée et de manière spontanée et très émotionnelle), tantôt des 
hommes et des institutions (aux grandes occasions et dans les sanctuaires, de façon plus rationnelle et organisée). À tous les niveaux, tous ces acteurs partagent le même système de croyances et de valeurs, celui de l'islam chiite pénétré de tradition iranienne.

L'idée d'une transaction liant le monde sacré au monde terrestre autour de la nourriture n'est évidemment propre ni à l'islam chiite ni à l'Iran. "Qui dit religion dit alimentation » (Goody 2006) : toute religion définit un certain rapport de la nourriture au sacré. La conception d'un échange avec le divin : « la pratique des offrandes alimentaires est un trait universel des liturgies religieuses " (Sabban 2010). Toutefois, il nous semble que le nazr en Iran met particulièrement en lumière le caractère interactif-l'intrication du don et du contre-don-et la double dimension terrestre et céleste que peut receler l'acte, en apparence si simple, de nourrir. Cette contribution espère donc apporter un nouvel élément à lépais dossier du lien entre nourriture et sacré en anthropologie. 
Sepideh Parsapajouh est anthropologue et chercheuse au CNRS au Centre d'études en sciences sociales du religieux (CéSor-EHESS). Sa première recherche a porté sur un bidonville iranien où elle a mis à jour un ordre fondé sur divers mécanismes de solidarité. Cette recherche l'a conduite à l'importance des systèmes de valeurs immatérielles et des croyances religieuses dans l'équilibre d'une société. Elle étudie aujourd'hui divers aspects de la religion chiite populaire, individuels et collectifs, intimes et spectaculaires, en Iran et ailleurs, en particulier les pratiques liées à la mort comme le culte des saints et des martyrs. Elle est auteur, entre autres, de : Au cour d'un bidonville Iranien, Paris, Karthala, 2016 ; "Sous le regard des martyrs à Téhéran ", l'Homme, $n^{\circ} 229,2019$, pp. 7-48; " La châsse de l'imam Husayn. Fabrique et parcours politique d'un objet religieux de Qom à Karbala ", Archives des sciences sociales des religions, $\mathrm{n}^{\circ} 174,2016$, pp. 49-74.

Emails : sepideh.parsapajouh@gmail.com ; sepideh.parsapajouh@ehess.fr

Sepideh Parsapajouh is an anthropologist and a CNRS research fellow at the Centre d'études en sciences sociales du religieux (CéSor-EHESS). Her first research focused on an Iranian slum where she uncovered an order based on various solidarity mechanisms. This research led her to the importance of immaterial value systems and religious beliefs in the balance of a society. Today, she studies various aspects of the popular Shiite religion, individual and collective, intimate and spectacular, in Iran and beyond, in particular practices related to death, devotional practices and the worship of saints and martyrs. She is the author, among others, of Au coeur d'un bidonville Iranien, Paris, Karthala, 2016; "Sous le regard des martyrs à Téhéran ", l'Homme, n 229, 2019, pp. 7-48; «La châsse de l'imam Husayn. Fabrique et parcours politique d'un objet religieux de Qom à Karbala ", Archives des sciences sociales des religions, $\mathrm{n}^{\circ} 174,2016$, pp. 49-74 (among others).

Emails: sepideh.parsapajouh@gmail.com; sepideh.parsapajouh@ehess.fr

\section{Notes}

1. Le 28 safar est la date de l'anniversaire de la mort du Prophète et du troisième imam selon le calendrier arabe lunaire.

2. Une sorte de riz au lait, parfumé au safran et à l'eau de rose.

3. Ce sont le Prophète Muhammad, sa fille Fâtemeh, son gendre et compagnon proche Ali, suivis des onze descendants de leur lignée, tous considérés comme purs de tout péché, d’où l'adjectif «impeccable » (ma’sûm) qu’on utilise pour les nommer.

4. Sha'bân, l'un des mois sacrés de l'islam pour les chiites. Ce mois est d'autant plus sacré qu'il est le mois des anniversaires de naissances de plusieurs imams, dont celui du Mahdi au 14 ${ }^{\text {ème }}$ jour connu sous le nom nimeh-ye Sha’bân (mi-Sha’bân). 
5. A ce sujet, voir Parsapajouh 2016 : 209-214; Bahar 2006.

6. Mais il y a aussi des nazr-s sans condition, non-intéressés, avec juste l'intention de se rapprocher d'un saint ou de Dieu.

7. Précisons toutefois que ce nazr matériel peut prendre aussi d'autres formes moins courantes, comme la construction d'un lieu de culte, d'une fontaine à boire, d'un saqqâa-khâneh (petit espace pour allumer des bougies votives), d'instruments et ustensiles pour les cérémonies de Moharram, etc.

8. Lieu de commémoration du martyre de l'imam Hosayn.

9. Pour plus d'exemples de ces nazr dans la littérature, voir Sharilu 2016.

10. A ce sujet en Iran, voir Vivier 2006 ; Arabestani 2020.

11. Le sperme est considéré comme particulièrement impur. Ainsi, tout individu adulte, après émission de semence, est dans un état d'impureté, appelé jenâbat (prononciation arabe : jinâba) : il est qualifié de jonob. Avant de reprendre sa vie religieuse, il doit procéder à grandes ablutions (ghosl-e jenâbat).

12. Appelé aussi la fête de la walâyat. Il s'agit du jour où le Prophète a solennellement désigné Ali comme son successeur au bord de l’étang de Ghadîr.

13. La sacralité de cet événement est liée au discours que l'imam Hosayn a prononcé ce jour, dans un lieu nommé 'arafa, avant de se diriger vers Karbala et d'y trouver le martyre.

14. Cette photo a été publié dans : S. Parsapajouh 2019 b.

15. Voir : https://shahraranews.ir/0001vw.

16. Pendant la pandémie de Covid-19, les volontaires continuent de préparer ce repas à Qom ; ils le distribuent dans des hôpitaux et auprès des nécessiteux (voir http:// hajj.ir/fa/99638).

17. Sur la notion de barzakh chez les théologiens et philosophes de l'Iislam, voir Terrier 2020.

18. Au sujet du repas pris au cimetière, voir aussi Fakouhi 2008.

\section{Bibliographie}

Arabestani, M. (2020), 'Cosmology, Identity and Food Related Rituals Among the Mandaeans', Archives des sciences sociales des religions : Religions en Iran, no. 189 : 203-226.

Bahar, M. 2004 (1383), 'Nazr va hoviyyat-e ejtemẩi-ye zanân' (Le don votif et l'identité sociale des femmes), Nâmeh-ye Olum-e Ejtemẩi (la revue La lettre des sciences sociales), no. $24: 17-132$.

Bromberger, Ch. (2013), Un autre Iran : Un ethnologue au Gilân (Paris : Armand Colin).

Fakouhi, N. (2008), 'Manger avec les morts : Pique-nique au cimetière en Iran', in Le pique-nique: Ou l'éloge d'un bonheur ordinaire, (dir.) F. Barthe-Deloizy, (Paris : Bréal), 92-105.

Mauss, M. [1924] (2009), 'Essai sur le don', in Sociologie et Anthropologie, (Paris : Presses universitaires de France).

Neuve-Eglise, A. (à paraitre), 'Modalités de l'extension d'une temporalité sacrée : la marche d'Arba'īn en Iran contemporain, entre logiques spirituelles et sociopolitiques'. 
Parsapajouh, S. (2016), Au cour d'un bidonville iranien (Paris : Karthala).

Parsapajouh, S. (2019), 'Les corps féminins et leurs expressions dans les cérémonies chiites de Ashura à Téhéran', in Etat-nation et fabrique du genre, des corps et des sexualités (Iran, Turquie et Afghanistan), (dir.) L. Direnberger et al., (Aix-enProvence : Presses universitaires de Provence), 49-72

Parsapajouh, S. (2020), “ La paradis de Zahra » : Le grand cimetière de Téhéran entre pratiques populaires et rationalité étatique', Revue Remmm : Cimetières et tombes dans les mondes musulmans. À la croisée des enjeux religieux, politiques et mémoriels, no. $146: 173-196$.

Ruhlmann, S. (2016), 'Méditation funéraire en Mongolie : Cercueil, yourte miniature et nourriture', Archives des sciences sociales des religions, numéro thématique : La force des objets-Matières à expériences, no. $174: 75-100$.

Sabban, F. (2010), 'Nourriture', in Dictionnaire des faits religieux, (éd.) D. Hervieu-Léger et R. Azria, (Paris : Presses universitaires de France), 801-808.

Sharilu, M. et Farzad, A. (2016) (1395), 'Anâsor farhang va konesh-e âmiyâneh dar dastand nevisân-e zan-e moâser' (Les éléments de la culture et d'action populaires dans les œuvres romancières contemporaines), Zan dar farhang va honar (le revue La femme dans la culture et l'art) 8, no. 1:1-22.

Soroushfar, Z. (2020), 'La gestion politique du calendrier par la République Islamique d'Iran', Archives des sciences sociales des religions : Religions en Iran, (dir.) S. Mervin et S. Parsapajouh, no. $189: 35-54$.

Terrier, M. (2020), 'La tombe comme isthme (barzakh) entre les vivants et morts : Points de vue croisés du soufisme et du shî̉isme imâmite (al-Ghazâlî et al-Fayd al-Kâshânî)', Revue Remmm : Cimetières et tombes dans les mondes musulmans. A la croisée des enjeux religieux, politiques et mémoriels, no. $146: 29-46$.

Vivier, A.-S. (2016), 'Le corps dans le chiisme populaire iranien : entre savâb et impureté', Revue Remmm, no. 113-114 : 125-149. 